\title{
Commentary \\ Low-molecular-weight cyclin E: the missing link between biology and clinical outcome
}

Said Akli and Khandan Keyomarsi

\author{
Department of Experimental Radiation Oncology, The University of Texas MD Anderson Cancer Center, Houston, Texas, USA \\ Corresponding author: Khandan Keyomarsi, kkeyomar@mdanderson.org \\ Published: 7 July 2004 \\ Breast Cancer Res 2004, 6:188-191 (DOI 10.1186/bcr905) \\ (c) 2004 BioMed Central Ltd
}

\begin{abstract}
Cyclin $E$, a key mediator of transition during the $G_{1} / S$ cellular division phase, is deregulated in a wide variety of human cancers. Our group recently reported that overexpression and generation of lowmolecular-weight (LMW) isoforms of cyclin $E$ were associated with poor clinical outcome among breast cancer patients. However, the link between LMW cyclin E biology in mediating a tumorigenic phenotype and clinical outcome is unknown. To address this gap in knowledge, we assessed the role of LMW isoforms in breast cancer cells; we found that these forms of cyclin E induced genomic instability and resistance to $p 21, p 27$, and antiestrogens in breast cancer. These findings suggest that high levels of LMW isoforms of cyclin $\mathrm{E}$ not only can predict failure to endocrine therapy but also are true prognostic indicators because of their influence on cell proliferation and genetic instability.
\end{abstract}

Keywords: antiestrogen resistance, breast cancer, genomic instability, low-molecular-weight cyclin E, prognostic marker

\section{Introduction}

Cell division relies on the activation of cyclins, which bind to their catalytic partners, the cyclin-dependent kinases (Cdks), to induce cell cycle progression from the $G_{0} / G_{1}$ phase to the $S$ phase and later to initiate mitosis. Cyclin $E$, a $G_{1}$ phase cyclin, has been shown to be overexpressed in many human cancers [1]. In tumor cells cyclin $E$ can be deregulated by a number of mechanisms: gene amplification [2,3], downregulation of p27 [4], and downregulation of its specific F-box protein Fbw7 (also called hCDC4), which tags phosphorylated cyclin E for proteosomal degradation [5-7]. Mutations in hCDC4 were found in breast, ovarian, endometrial [8] and colorectal cancers [6], and were associated with elevated levels of cyclin $E$ protein. Moreover, cyclin $E$ overexpression in breast epithelium predisposes transgenic mice to neoplasia [9]. A new mechanism of cyclin $E$ deregulation recently described by our group is the proteolytic processing of full-length cyclin $E$ at two sites in the amino-terminus by an elastase-like protease, independently of proteasome-mediated proteolytic degradation $[10,11]$.
The predominant $50-k D a$ isoform of cyclin $\mathrm{E}$, called $\mathrm{EL} 1$, is found in both normal cells and tumor cells, and it is encoded by an alternative, spliced mRNA that is different from originally cloned cDNA [11,12]. The EL4 cyclin E isoform is accounted for by alternative translation at methionine 46, whereas cleavage at two distinct sites in the amino-terminus accounts for the two pairs of lowmolecular-weight (LMW) cyclin $E$ isoforms (EL2/3 and EL5/6). Only tumor cells have the machinery to process cyclin E into its LMW forms [10]. These LMW isoforms are nuclear and functionally active because they increase the progression from $G_{1}$ to $S$ phase [11].

Overexpression of the LMW forms in immortalized mammary epithelial cells resulted in decreased doubling time and increased cyclin $E$ kinase activity [13]. Additionally, overexpression of these hyperactive cyclin $\mathrm{E}$ isoforms in ovarian cells deregulated the $G_{1} / S$ transition [14]. The prognostic potential of LMW isoforms of cyclin $E$ was recently demonstrated in a retrospective study of 395 breast cancer patients; the LMW isoforms were found to be much more powerful predictors of poor outcome than 
were estrogen and progesterone receptor status or levels of cyclin $D_{1}$, cyclin $D_{3}$, and HER2/neu [15]. On multivariate analysis, cyclin $\mathrm{E}$ was found to be eight times more predictive of poor prognosis than was nodal status [15].

Recently, we directly assessed the mechanism by which the LMW forms of cyclin E contribute to breast tumor cell progression [16]. In this commentary we highlight biochemical and functional differences between the fulllength and LMW isoforms of cyclin E, which may help to explain how LMW cyclin $E$ isoforms are strong predictors of breast cancer mortality. This work provides the link between the prognostic power of LMW cyclin $\mathrm{E}$ isoforms and their biology in breast cancer.

\section{Low-molecular-weight cyclin $E$ and resistance to $p 21$ and p27 inhibition}

Constitutive overexpression of cyclin $E$ has been shown to shorten the duration of the $G_{1}$ phase, to decrease cell size, and to diminish requirements for growth factors $[12,17]$. In addition, cyclin $\mathrm{E}$ overexpression has been shown to be associated with increased resistance to transforming growth factor- $\beta$ mediated growth inhibition in some cell lines [18], delayed response to lovastatininduced $G_{1}$ arrest in NIH3T3 cells [19], and partially abrogated tamoxifen-induced G1 arrest in MCF-7 cells $[20,21]$. The common mediators of these growth inhibitory signals are Cdk inhibitors, which ultimately inhibit cyclin $\mathrm{E}$ and Cdk2 kinase activity.

To assess the sensitivity of LMW isoforms of cyclin $E$ to Cdk inhibition, we stably transfected MCF-7 cells with constructs encoding the full-length and two LMW forms representing elastase-cleaved variants of cyclin E [16]. Clones with equal levels of cyclin E overexpression (fivefold greater than the endogenous expression) were selected for comparison. Cyclin E overexpression led to induction of $p 53$ and upregulation of $p 21$, which partially abrogated cyclin $E$ and Cdk2 kinase activity of the full-length form of cyclin E. Despite having the same levels of protein overexpression as the full-length form, the LMW forms of cyclin $\mathrm{E}$ had twofold greater associated kinase activity. This was not due to a defect in p21 or p27 binding, as was shown by immunoprecipitation followed by Western blot analysis. This conclusion was further confirmed when we used the baculovirus expression system, which allowed for precise control of the expression (i.e. amount) of the different proteins implicated in the activity of the cyclin $\mathrm{E}$ and Cdk2 complexes. In this system, the $\mathrm{IC}_{50}(50 \%$ inhibitory concentration) of $p 21$ required to inhibit the LMW forms of cyclin $\mathrm{E}$ was three to fivefold greater than that needed to inhibit the full-length form; similar results were obtained with $p 27$.

In a parallel study using an ovarian cancer cell line model, our group demonstrated an increased resistance to lovastatin induced $G_{1}$ arrest in cells overexpressing the LMW isoforms of cyclin $E$, as compared with cells not expressing these forms, despite the binding of $p 21$ and p27 to these LMW isoforms [14]. Removing 40-69 amino acids from the amino-terminus of cyclin $E$ conferred new functions to these deleted isoforms, which resisted the action of cell cycle inhibitors and extended the range of Cdk substrates. Because Cdk inhibitors are the mediators of antiestrogen-induced cell growth inhibition, these observations prompted us to explore the role of LMW cyclin $\mathrm{E}$ in antiestrogen resistance.

\section{Low-molecular-weight cyclin $E$ and resistance to antiestrogen treatment}

To decipher the effect of cyclin $E$ overexpression on antiestrogen sensitivity, the pure antiestrogen ICl 182,780 was added to MCF-7 cells to block estrogen receptor signaling, and the $\mathrm{S}$ phase fraction was measured by flow cytometry at different times after addition of the drug. These experiments showed that cells transfected with LMW cyclin $E$ were more refractory to $I C l$-induced $G_{1}$ arrest than were cells transfected with the full-length form over a 3-day period. This increased resistance was shown to be due to decreased sensitivity of LMW cyclin $E$ associated kinase activity to $p 21$ and $p 27$ inhibition.

These experiments confirm that overexpression of fulllength cyclin $E$ results in only a slight decrease in sensitivity to the growth-inhibiting effects of antiestrogens $[20,21]$; these results also demonstrate that LMW cyclin E overexpression results in sustained resistance.

To corroborate our in vitro data, we tested the hypothesis that patients with ER positive tumors who were eligible for adjuvant systemic therapy including hormonal therapy would not benefit from antiestrogen treatment if their tumors had high levels of total cyclin E. Among 150 stage I-III estrogen receptor-positive patients who had high levels of cyclin $E$, we found no difference in diseasespecific survival rates between patients receiving antiestrogen treatment with those not treated with antiestrogens ( $P=0.083$, log rank test). This observation suggests that tumors that greatly overexpress cyclin $E$ are resistant to the effects of antiestrogen therapy. An independent study also found that cyclin $\mathrm{E}$ is a strong predictor of endocrine therapy failure in human breast cancer [22].

\section{Low-molecular-weight cyclin E and genomic instability}

Constitutive overexpression of cyclin $E$ in both immortalized rat embryo fibroblasts and human breast epithelial cells induces chromosomal instability without changing centrosome numbers but while impairing $S$ phase progression, pointing to possible alterations in the process of licensing the origins of DNA replication [23]. 
Additionally, the overexpression and appearance of LMW cyclin E are associated with microsatellite instability in colorectal carcinomas [24].

In our studies we pinpointed that the LMW forms of cyclin $\mathrm{E}$ and, to a lesser extent, the full-length forms were the mediators of genomic instability. It should be noted that in all previous studies $[23,24]$ the form of cyclin E used was an alternatively spliced cyclin $E$ that did not give rise to protein products in cell lines or tissues. For our analysis we used constructs representing the fulllength and the LMW forms of cyclin E. We observed that although overexpression of cyclin $E$ isoforms increased the fraction of cells in $S$ phase, it did not decrease the doubling time of the cells, suggesting an $S$ phase delay. However, flow cytometry analysis demonstrated a four to sixfold increase in the percentage of cells with polyploid DNA. Karyotype analysis revealed a four to sevenfold increase in the number of chromosome aberrations in those cells that overexpressed the LMW forms. These aberrations included multiple chromosomal fragments and subtelomeric chromatid breaks, resulting in telomeric associations. In addition, a three to fourfold increase in the number of polyploid and tetraploid cells was observed in LMW cyclin E transfectants, most likely as a result of chromosomal endoreduplication. We also found a significant correlation $(P=0.0003)$ between ploidy and cyclin E protein levels among 331 stage I-III breast cancer patients. Of patients whose tumors expressed high levels of cyclin E, the 5-year disease specific survival rate was significantly lower among those with polyploid tumors $(P=0.02$, log-rank test). These results suggest that overexpression of the LMW forms of cyclin $\mathrm{E}$ induced genomic instability by promoting aneuploidy and the formation of structural chromosomal lesions.

These observations indicate that cyclin $\mathrm{E}$ overexpression interferes with the processes shown to be affected when cyclin $E$ is deleted in mice, such as occurs in endoreduplication and firing of the origin of replication. Cyclin $\mathrm{E}_{1} / \mathrm{E}_{2}$ double knockout mice died during mid-gestation because of impairment of endoreduplication in the trophoblast giant cells of the placenta [25]. In addition, cyclin $E$ null mouse embryonic fibroblasts could not exit from $G_{0}$ because of the absence of MCM2 loading on the origin of replication. Most importantly, cyclin E knockout mouse embryonic fibroblasts were resistant to oncogenic transformation, confirming the crucial role played by cyclin $\mathrm{E}$ in tumorigenesis.

The phenotype of the Cdk2 knockout mice, which were viable and had no developmental defects, also raised questions about the function of cyclin $\mathrm{E}$ independent of Cdk2 [26,27] and p27 binding [28]. Recent work also
CDC4 gene, cyclin E overexpression, and genomic instability, excluding the implication of other biologic processes in this phenomenon [6].

\section{Conclusion}

In summary, overexpression of LMW cyclin E isoforms in tumor cells conferred biologic properties that impacted on the cells' resistance to cell growth inhibitory signals and on processes that led to genomic instability, such as DNA replication and chromosomal segregation. The increased sensitivity of LMW cyclin E overexpressing cells to genomic instability could help to explain why these isoforms are such strong predictors of poor outcome among patients with node negative, stage I breast cancer. Once expressed, these forms drive the cells to a more aggressive phenotype, and their detection at such an early stage, especially in patients with negative lymph nodes, could help physicians to make a decision toward more aggressive treatments. This study implies that estrogen receptor positive tumors that express high levels of LMW cyclin $\mathrm{E}$ will respond poorly to antiestrogens; thus, patients with these tumors should be considered for alternative therapeutic strategies. These data could be used to find inhibitors of LMW isoforms of cyclin $E$ that could specifically target and kill tumor cells. We showed in this study that high levels of LMW cyclin E isoforms not only can predict failure of endocrine therapy but also are true prognostic indicators because of their influence on cell proliferation and genetic instability.

\section{Competing interests}

None declared.

\section{Acknowledgments}

This work was supported in part by grant (R01-CA87548) from the National Cancer Institute to KK and the MDACC Cancer Center Grant P30-CA16672 from the National Institute of Health. SA is supported in part by the National Institutes of Health SPORE Grant P50 CA91846.

\section{References}

1. Akli S, Keyomarsi K: Cyclin E and its low molecular weight forms in human cancer and as targets for cancer therapy. Cancer Biol Ther 2003, Suppl 1:S38-S47.

2. Cassia R, Moreno-Bueno G, Rodriguez-Perales S, Hardisson D, Cigudosa JC, Palacios J: Cyclin E gene (CCNE) amplification and hCDC4 mutations in endometrial carcinoma. J Pathol 2003, 201:589-595.

3. Schraml $P$, Bucher $C$, Bissig $H$, Nocito A, Haas $P$, Wilber $K$ Seelig S, Kononen J, Mihatsch MJ, Dirnhofer S, et al.: Cyclin E overexpression and amplification in human tumours. $J$ Pathol 2003, 200:375-382.

4. Bloom J, Pagano M: Deregulated degradation of the cdk inhibitor p27 and malignant transformation. Semin Cancer Biol 2003, 13:41-47.

5. Koepp DM, Schaefer LK, Ye X, Keyomarsi K, Chu C, Harper JW, Elledge SJ: Phosphorylation-dependent ubiquitination of cyclin E by the SCFFbw7 ubiquitin ligase. Science 2001, 294:173-177.

6. Rajagopalan $\mathrm{H}$, Jallepalli PV, Rago C, Velculescu VE, Kinzler KW, Vogelstein B, Lengauer C: Inactivation of hCDC4 can cause chromosomal instability. Nature 2004, 428:77-81.

7. Strohmaier H, Spruck $\mathrm{CH}$, Kaiser P, Won KA, Sangfelt O, Reed SI: Human F-box protein hCdc4 targets cyclin E for proteolysis and is mutated in a breast cancer cell line. Nature 2001, 413:316-322. 
8. Spruck $\mathrm{CH}$, Strohmaier $\mathrm{H}$, Sangfelt $\mathrm{O}$, Muller HM, Hubalek M, Muller-Holzner E, Marth C, Widschwendter M, Reed SI: hCDC4 gene mutations in endometrial cancer. Cancer Res 2002, 62: 4535-4539.

9. Bortner DM, Rosenberg MP: Induction of mammary gland hyperplasia and carcinomas in transgenic mice expressing human cyclin E. Mol Cell Biol 1997, 17:453-459.

10. Harwell RM, Porter DC, Danes C, Keyomarsi K: Processing of cyclin $\mathrm{E}$ differs between normal and tumor breast cells. Cancer Res 2000, 60:481-489.

11. Porter DC, Zhang N, Danes C, McGahren MJ, Harwell RM, Faruki $\mathrm{S}$, Keyomarsi K: Tumor-specific proteolytic processing of cyclin E generates hyperactive lower-molecular-weight forms. Mol Cell Biol 2001, 21:6254-6269.

12. Ohtsubo M, Theodoras AM, Schumacher J, Roberts JM, Pagano $M$ : Human cyclin E, a nuclear protein essential for the G1-to-S phase transition. Mol Cell Biol 1995, 15:2612-2624.

13. Wingate $\mathrm{H}$, Bedrosian I, Akli S, Keyomarsi K: The low molecular weight (LMW) isoforms of cyclin $E$ deregulate the cell cycle of mammary epithelial cells. Cell Cycle 2003, 2:461-466.

14. Bedrosian I, Lu KH, Verschraegen C, Keyomarsi K: Cyclin E deregulation alters the biologic properties of ovarian cancer cells. Oncogene 2004, 23:2648-2657.

15. Keyomarsi K, Tucker SL, Buchholz TA, Callister M, Ding Y, Hortobagyi GN, Bedrosian I, Knickerbocker C, Toyofuku W, Lowe M, et al.: Cyclin $\mathrm{E}$ and survival in patients with breast cancer. $N$ Engl $J$ Med 2002, 347:1566-1575.

16. Akli S, Zheng P-J, Multani AS, Wingate HF, Pathak S, Zhang N, Tucker SL, Chang S, Keyomarsi K: Tumor-specific low-molecular-weight forms of cyclin $E$ induce genomic instability and resistance to p21, p27, and antiestrogens in breast cancer. Cancer Res 2004:in press.

17. Ohtsubo M, Roberts JM: Cyclin-dependent regulation of G1 in mammalian fibroblasts. Science 1993, 259:1908-1912.

18. Sgambato A, Doki Y, Schieren I, Weinstein IB: Effects of cyclin E overexpression on cell growth and response to transforming growth factor beta depend on cell context and p27Kip1 expression. Cell Growth Differ 1997, 8:393-405.

19. Ghosh PM, Moyer ML, Mott GE, Kreisberg Jl: Effect of cyclin E overexpression on lovastatin-induced G1 arrest and RhoA inactivation in NIH3T3 cells. J Cell Biochem 1999, 74:532-543.

20. Dhillon NK, Mudryj M: Ectopic expression of cyclin E in estrogen responsive cells abrogates antiestrogen mediated growth arrest. Oncogene 2002, 21:4626-4634.

21. Hui R, Finney GL, Carroll JS, Lee CS, Musgrove EA, Sutherland $\mathrm{RL}$ : Constitutive overexpression of cyclin D1 but not cyclin $\mathrm{E}$ confers acute resistance to antiestrogens in T-47D breast cancer cells. Cancer Res 2002, 62:6916-6923.

22. Span PN, Tjan-Heijnen VC, Manders P, Beex LV, Sweep CG: Cyclin-E is a strong predictor of endocrine therapy failure in human breast cancer. Oncogene 2003, 22:4898-4904.

23. Spruck $\mathrm{CH}$, Won KA, Reed SI: Deregulated cyclin E induces chromosome instability. Nature 1999, 401:297-300.

24. Sutter T, Dansranjavin T, Lubinski J, Debniak T, Giannakudis J, Hoang-Vu C, Dralle H: Overexpression of cyclin E protein is closely related to the mutator phenotype of colorectal carcinoma. Int J Colorectal Dis 2002, 17:374-380.

25. Geng Y, Yu Q, Sicinska E, Das M, Schneider JE, Bhattacharya S, Rideout WM, Bronson RT, Gardner H, Sicinski P: Cyclin E ablation in the mouse. Cell 2003, 114:431-443.

26. Ortega S, Prieto I, Odajima J, Martin A, Dubus $P$, Sotillo $R$ Barbero JL, Malumbres M, Barbacid M: Cyclin-dependent kinase 2 is essential for meiosis but not for mitotic cell division in mice. Nat Genet 2003, 35:25-31.

27. Berthet C, Aleem E, Coppola V, Tessarollo L, Kaldis P: Cdk2 knockout mice are viable. Curr Biol 2003, 13:1775-1785.

28. Geisen $C$, Moroy $T$ : The oncogenic activity of cyclin $E$ is not confined to Cdk2 activation alone but relies on several other, distinct functions of the protein. J Biol Chem 2002, 277: 39909-39918. 